Prolyl oligopeptidase inhibition attenuates the toxicity of a proteasomal inhibitor, lactacystin, in the alpha-synuclein overexpressing cell culture

\title{
Myöhänen, Timo T.
}

2017-01-01

Myöhänen , T T , Norrbacka , S \& Savolainen , M H 2017 , ' Prolyl oligopeptidase inhibition attenuates the toxicity of a proteasomal inhibitor, lactacystin, in the alpha-synuclein overexpressing cell culture ' , Neuroscience Letters , vol. 636 , pp. 83-89 . https://doi.org/10.1016/j.neulet.2016.11.00

http://hdl.handle.net/10138/307680

https://doi.org/10.1016/j.neulet.2016.11.008

cc_by_nc_nd

acceptedVersion

Downloaded from Helda, University of Helsinki institutional repository.

This is an electronic reprint of the original article.

This reprint may differ from the original in pagination and typographic detail.

Please cite the original version. 


\section{Prolyl oligopeptidase inhibition attenuates the toxicity of a proteasomal} inhibitor, lactacystin, in the alpha-synuclein overexpressing cell culture

Timo T. Myöhänen*, Susanna Norrbacka and Mari H. Savolainen

Division of Pharmacology and Pharmacotherapy, Faculty of Pharmacy

Viikinkaari 5E (P.O.Box 56), 00014 University of Helsinki, FINLAND

emails: timo.myohanen@helsinki.fi, susanna.norrbacka@helsinki.fi,

mari.x.savolainen@helsinki.fi

Corresponding author: Dr. Timo T. Myöhänen, $\mathrm{PhD}$

Division of Pharmacology and Pharmacotherapy

Viikinkaari 5E (P.O.Box 56), FIN-00014 University of Helsinki, FINLAND

1

\footnotetext{
${ }^{1}$ Abbreviations: aSyn, alpha-synuclein; cell lines stably overexpressing human A53T mutated form of aSyn, [A53T]aSyn or A30P mutated form of aSyn, [A30P]aSyn; high-molecularweight, HMW; microtubule-associated protein light chain 3B I-II, LC3BI-II; negative control, NC; optical density, OD; Parkinson's disease, PD; prolyl oligopeptidase, PREP; ubiquitinproteasome system, UPS; Western blot, WB; Wild type, Wt.
} 


\begin{abstract}
Lewy bodies, the histopathological hallmarks of Parkinson's disease (PD), contain insoluble and aggregated $\alpha$-synuclein (aSyn) and many other proteins, proposing a role for failure in protein degradation system in the PD pathogenesis. Proteasomal dysfunction has indeed been linked to PD and aSyn oligomers have been shown to inhibit proteasomes and autophagy. Our recent studies have shown that inhibitors of prolyl oligopeptidase (PREP) can prevent the aggregation and enhance the clearance of accumulated aSyn, and therefore, we wanted to study if PREP inhibition can overcome the aSyn aggregation and toxicity induced by lactacystin, a proteasomal inhibitor. The cells overexpressing human A30P or A53T mutated aSyn were incubated with lactacystin and a PREP inhibitor, KYP-2047, for $48 \mathrm{~h}$. Theafter, the cells were fractioned, and the effects of lactacystin with/without $1 \mu \mathrm{M}$ KYP-2047 on aSyn aggregation and ubiquitin accumulation, cell viability and on autophagic markers (p62, Beclin1 and LC3BII) were studied. We found that KYP-2047 attenuated lactacystin-induced cell death in mutant aSyn overexpressing cells but not in non-overexpressing control cells. KYP-2047 reduced significantly SDS-insoluble high-molecular-weight aSyn oligomers that was in line with the cell viability results. In addition, significant reduction in protein accumulation marker, p62, was seen in SDS fraction while LC3BII, a marker for autophagosome formation, was increased, indicating to enhanced autophagy. Our results further streghten the possibilities for PREP inhibitors as a potential drug therapy against synucleinopathies and other protein aggregating diseases.
\end{abstract}

Keywords: Serine protease, Parkinson's disease, synucleinopathies, alpha-synuclein aggregation, enzyme inhibition 


\section{Introduction}

In Parkinson's disease (PD), degeneration of dopaminergic neurons in substantia nigra and striatum leads to progressing movement deficits. The cause for this neurodegeneration is not known but histopathological findings of PD are insoluble inclusions, Lewy bodies, mainly consisting of an $\alpha$-synuclein (aSyn) [1].

aSyn is a natively unfolded protein that can misfold, leading to protein aggregation, formation of toxic oligomers and cell-to-cell propagation of aSyn pathology [2-4]. Moreover, genomewide association studies have pointed aSyn gene, $S N C A$, as a risk factor for PD and mutations in $S N C A$ are associated with familial forms of $\mathrm{PD}$, supporting its participation in PD pathology (for review, see [5]). The most studied point mutations in SNCA are A30P and A53T, causing familial PD with early onset. Both of them enhance the fibrillization of aSyn, leading to aSyn oligomers and cellular damages, but A30P is more prone for membrane-induced aggregation (for review, see, [6]). In addition to damaging several cellular organelles, aSyn overexpression and aSyn oligomers have been shown to inhibit ubiquitin-proteasome system (UPS) [7,8], and decreased proteasomal activity in the substantia nigra has been identified in PD patients [9]. Moreover, aSyn can inhibit and impair chaperone-mediated autophagy [10] and macroautophagy (referred as autophagy hereafter) [11], thus inducing its own accumulation. In addition, several reports show that proteasomal inhibition induces the aggregation of aSyn in cells and in vivo [12-14], stressing the importance of proteolytic balance in protein aggregation diseases.

Several factors can induce and enhance the aggregation process of aSyn (for review, see [4]). On of them is prolyl oligopeptidase (PREP), a serine protease that can increase the aggregation of aSyn via direct protein-protein interaction $[15,16]$. PREP is also colocalized with aSyn in substantia nigra of post mortem samples of PD patients, supporting the role of this interaction 
in PD pathology [17]. Recently, we have shown that inhibition of PREP by a small-molecule inhibitor reduces aSyn protein aggregation in vitro and in vivo by modifying the interaction between PREP and aSyn [15,18-20]. Moreover, we have shown that PREP is a negative regulator of autophagy and that PREP inhibition enhances the autophagic flux via beclin1 dependent manner [19], leading to decreased amount of high-molecular weight (HMW) aSyn oligomers in cells and in aSyn transgenic mouse models.

Several studies suggest that when proteasomal processing of the proteins is impaired, autophagy becomes important in degradation of accumulated proteins (for review, see [21]). Moreover, induction of autophagy is shown attenuate the toxicity of aSyn aggregation (for review, see [21]). Thus, based on our previous findings, we wanted to test whether inhibition of PREP is protective against a proteasomal inhibitor, lactacystin, induced toxicity and protein accumulation in aSyn overexpressing cell culture.

\section{Materials and methods}

\section{Chemicals}

Chemicals used were purchased form Sigma-Aldrich (St. Louis, MO, USA) unless otherwise specified. Ethanol was purchased from Altia (Helsinki, Finland). Lactacystin was purchased from A.G. Scientific (\#L-1147; San Diego, CA, USA) and dissolved in PBS in concentration of $2 \mathrm{mg} / \mathrm{ml}$. The PREP inhibitor, KYP-2047 (4-phenylbutanoyl-1-prolyl-2(S)cyanopyrrolidine), was synthesized in School of Pharmacy, University of Eastern Finland, as previously described [22]. KYP-2047 has been shown to be highly potent, brain and cell penetrating and specific for PREP [23,24]. KYP-2047 was dissolved in DMSO at a concentration of $100 \mathrm{mM}$ for stock. 


\section{Cell lines}

Stable cell lines overexpressing human A53T ([A53T]aSyn) or A30P aSyn ([A30P]aSyn) were generated using a lentiviral vector as described in [25]. SH-SY5Y human neuroblastoma cell line without aSyn overexpression (Wt cells) was purchased from ATCC (LGC Standards; \#CRL-2266, Middlesex, UK). Cells were cultured with Dulbecco's modified eagle medium (DMEM-Glutamax, \#31966-021; ThermoFisher, Waltham, MA, USA) containing 15\% fetal bovine serum (FBS, \#16000-044; ThermoFisher), 1\% non-essential amino acids (NEAA, \#11140; ThermoFisher) and $50 \mu \mathrm{g} / \mathrm{ml}$ Gentamycin (\#15750-094; ThermoFisher). With aSyn overexpressing cells $1 \mu \mathrm{g} / \mathrm{ml}$ puromycin was added to medium for selection [18].

\section{Induction of aSyn aggregation by lactacystin, a proteasomal inhibitor}

For Western blot (WB) and proteasomal activity experiments, the cells were seeded in a 6-well plate with the density 400,000 cells/well and allowed to attach overnight. Thereafter, the cells were incubated for $24 \mathrm{~h}$ on $10 \mu \mathrm{M}$ lactacystin with $1 \mu \mathrm{M}$ KYP-2047 or $0.001 \%$ DMSO (vehicle control). The concentration of KYP-2047 was chosen based on earlier studies $[18,19]$ and on dose-response experiment (Fig. S1A in supplementary material). Lactacystin dose was chosen based on cell viability results on dose-response experiments (Fig. 1B-D). After the incubation, the cells were homogenized as described below.

\section{Proteasome activity assay}

For determining chymotrypsin-like 20S proteasomal activity, the protocol based on Suc-LeuLeu-Val-Tyr-AMC (\#I-1395, Bachem, Bubendorf, Switzerland) substrate was used as described in [19]. The fluorescence was read with Victor 2 well-plate reader (PerkinElmer, Waltham, MA, USA) and all assays were done in triplicate.

\section{Cell viability assay}


Cells were plated with the density of 50,000 cell/well in 96 -well plate and the next day incubated for $24 \mathrm{~h}$ with variable concentrations of lactacystin $(10 \mathrm{nM}, 100 \mathrm{nM}, 1 \mu \mathrm{M}, 10 \mu \mathrm{M}$ and $100 \mu \mathrm{M})$ in the presence of $1 \mu \mathrm{M} \mathrm{KYP}-2047$ or DMSO vehicle $(0.001 \%$ DMSO; 150 $\mu \mathrm{L} /$ well). To assess cell viability a standard LDH release assay was performed as previously described [18].

\section{Cell fractionation and WB}

WB was used to detect aSyn, ubiquitin accumulation and autophagy markers from cellular fractions. The cells were fractioned to TBS soluble (soluble aSyn), Triton X-100 soluble (membrane-bound aSyn) and SDS soluble (containing SDS soluble and insoluble aSyn conformations) fractions as described in [26]. Before WB, the protein concentrations of TBS and Triton X-100 fractions were measured by using BCA method (Bio-Rad, Hercules, CA, USA), and the samples were loaded to $4-20 \%$ TGX gels (Bio-Rad) with equal protein amounts. The loading of SDS-fraction was based on protein amount of triton X-100 soluble fraction. Standard SDS-PAGE techniques were used, and the membranes were incubated $+4{ }^{\circ} \mathrm{C}$ overnight in 5\% skim milk in $0.05 \%$ Tween 20 in TBS. Following primary antibodies were used: mouse anti-aSyn (1:1000; \#ab1903, AbCam), mouse anti-ubiquitin (1:1000; \#3936S, Cell Signaling Technology, Danvers, MA, USA), mouse anti-SQSTM1/p62 (p62, 1:5000; \#ab56416, AbCam), rabbit anti-microtubule associated protein light chain 3B I-II (LC3BI-II, 1:1000; \#L7843), rabbit anti-beclin1 (1:2000; \#ab16998, AbCam) and rabbit anti- $\beta$-actin (loading control, 1:2000; \#4967S, Cell Signaling Technology). After overnight incubation, the membranes were washed and incubated with appropriate HRP-conjugated secondary antibodies for $2 \mathrm{~h}$ in room temperature [for aSyn and SQSTM1/p62, goat anti-mouse HRP (dilution 1:2000 in 5\% milk, \#31430; Thermo Fischer); for ubiquitin, LC3BI-II, beclin1, parkin and $\beta$-actin, goat anti-rabbit (dilution 1:2000; Product \#31463, Thermo Fisher Scientific)]. The images were captured using the C-Digit imaging system (Licor, Lincoln, NE, USA). Three independent WB 
experiments were performed. ImageJ was used for analysing bands, and the optical density (OD) value was calculated by comparing the OD value to the corresponding beta-actin OD value.

\section{Statistics}

Statistical analysis was done using GraphPad Prism 6 software (GraphPad Software, La Jolla, CA, USA) and SPSS 21 (cell viability; IBM Analytics, Armonk, NY, USA). 1-way analysis of variance (ANOVA) with Newman-Keuls post-hoc test or 2-way ANOVA with Bonferroni posthoc test were used. For cell viability data, 2-way ANOVA (general difference between treatments), and t-test (difference between treatments in different concentrations) were performed. Data is presented as Mean \pm SEM and $\mathrm{P}<0.05$ was considered as statistically significant.

\section{Results}

\section{The effect of lactacystin and KYP-2047 on cell viability}

A $24 \mathrm{~h}$ lactacystin incubation $(10 \mu \mathrm{M})$ decreased the $20 \mathrm{~S}$ proteasomal activity $68 \%$ (Wt cells) to $75 \%$ ([A53T]aSyn and [A30P]aSyn cells) (Fig. 1A; $\mathrm{P}=0.0002 ; \mathrm{F}_{1,6}=67.78,2$-way ANOVA). $1 \mu \mathrm{M}$ KYP-2047 had no significant effects on proteasomal activity (Fig. S1B in supplementary material) and aSyn overexpression did not alter the basal proteasomal activity (Fig. 1A). Lactacystin induced cell death assayed by LDH test dose-dependently on both aSyn overexpressing cell lines (Fig. 1C-D; [A53T]aSyn, $\mathrm{P}<0.0001$; [A30P]aSyn, $\mathrm{P}<0.0001$, 2-way ANOVA) but not in Wt cells without aSyn overexpression (Fig. 1B). KYP-2047 significantly reduced the lactacystin toxicity on both aSyn cell lines (Fig. 1C-D; [A53T]aSyn, P=0.0121, $\mathrm{F}_{1,24}=7.370 ;$ [A30P]aSyn, $\mathrm{P}<0.0114, \mathrm{~F}_{1,24}=7.517,2$-way ANOVA), being the most significant 
at $10 \mu \mathrm{M}$ lactacystin dose (Fig. 1C-D; [A53T]aSyn, $\mathrm{P}=0.048$; [A30P]aSyn, $\mathrm{P}=0.05$, t-test), corresponding the reduction seen on aSyn oligomers with same lactacystin dose (Fig. 2).
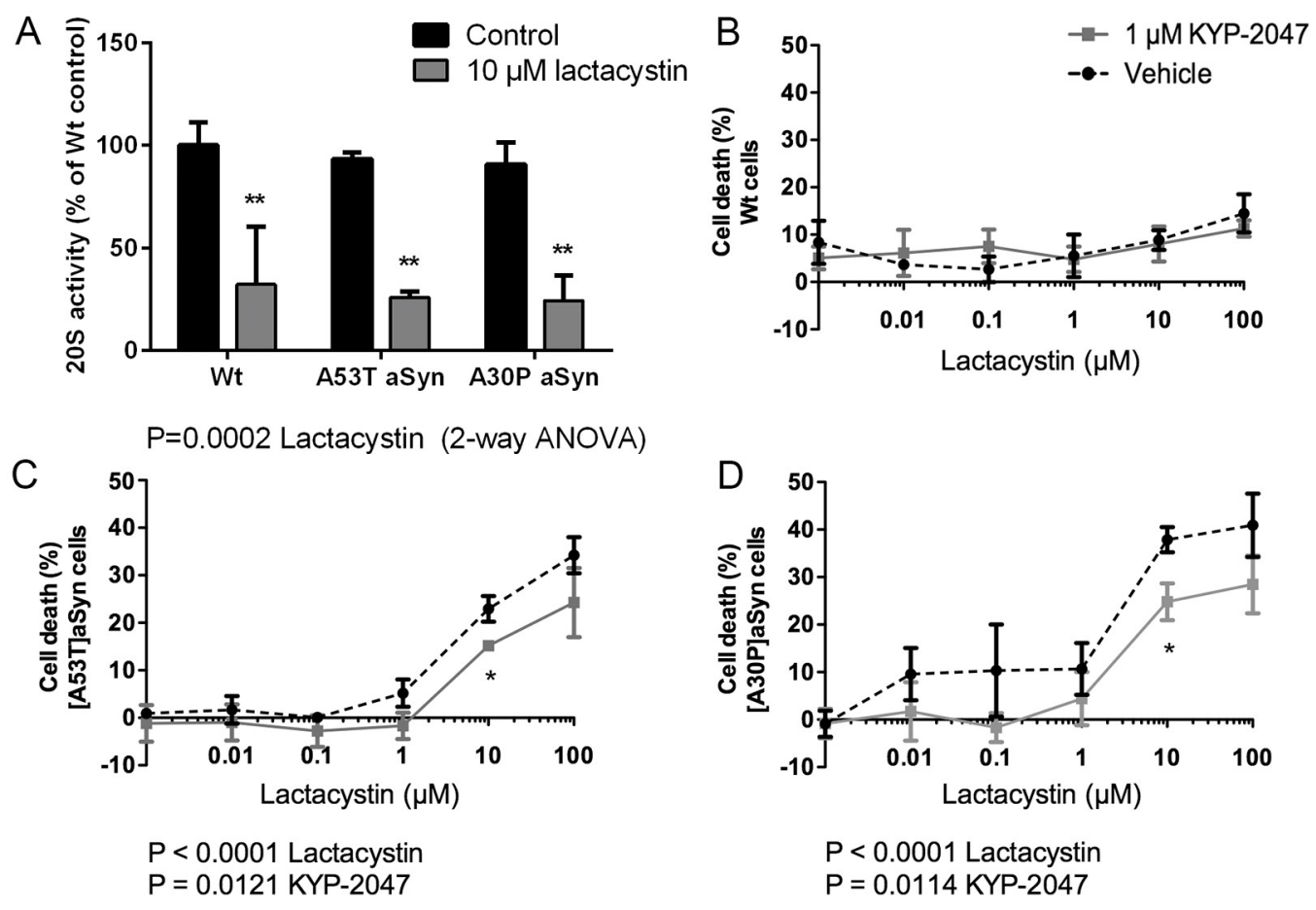

Figure 1A-D. The effect of $24 \mathrm{~h}$ lactacystin (LC) incubation (10 $\mu \mathrm{M})$ on $20 \mathrm{~S}$ proteasomal activity and on cell viability of wild-type SH-SY5Y cells (Wt) and [A53T]aSyn and [A30P]aSyn overexpressing cells. LC significantly decreased the 20S proteasomal activity (A), while $\alpha$-synuclein (aSyn) overexpression did not affect the basal proteasomal activity or sensitivity for LC. $24 \mathrm{~h}$ incubation with LC (0.01-100 $\mu \mathrm{M})$ had no effect on cell viability of nonoverexpressing Wt cells (B) but was significantly toxic to aSyn overexpressing cells $(\mathrm{P}<$ 0.0001, 2-way ANOVA). KYP-2047 $(1 \mu \mathrm{M})$ reduced the toxicity caused by LC on aSyn overexpressing cells $(\mathrm{P}=0.0121$ in [A53T]aSyn cells; $\mathrm{P}=0.0114$ in [A30P]aSyn cells; 2-way ANOVA). The effect was the most evident in $10 \mu \mathrm{M}$ lactacystin dose (C-D). * P $<0.05 \mathrm{KYP}-$ 2047 vs. vehicle, t-test; $* * \mathrm{P}<0.01$, vs control, Bonferroni post-hoc test, 2-way ANOVA.

\section{The effect of PREP inhibition on lactacystin-induced aSyn aggregation}

Lactacystin incubation increased the levels of aSyn dimers (37 kDA band) but did not have an effect on the levels of higher molecular weight aSyn forms in TBS fraction of either cell line (Fig. 2A), but increased the levels of Triton-X soluble oligomers in [A30P]aSyn cells by 114 
(vehicle) and 153\% (KYP-2047) (Fig. 2B; $\mathrm{P}=0.0416 ; \mathrm{F}_{2,8}=5.655$, 1-way ANOVA). aSyn oligomers in SDS-fraction were increased in both aSyn overexpressing cell lines but significant increase compared to control was seen only in [A30P]aSyn cells (Fig. 2C; $\mathrm{P}=0.0194$; $\mathrm{F}_{2,9}=6,308,1$-way ANOVA; $\mathrm{P}<0.05$ negative control (NC) vs. lactacystin+vehicle, NewmanKeuls post-hoc test). In [A30P]aSyn cells, KYP-2047 significantly decreased the levels of lactacystin-induced aSyn oligomers in SDS fraction (93\% reduction compared to vehicle) (Fig. 2C; $P<0.05$ lactacystin+vehicle vs. lactacystin+KYP-2047, Newman-Keuls post-hoc test) and similar trend was seen in the SDS fraction of [A53T]aSyn cells. Lactacystin incubation did not result in aSyn oligomerazion in wildtype SH-SY5Y cells without aSyn overexpression and monomeric form of aSyn was not changed in any of the fractions (see Fig. S2 and S3 in supplementary material).

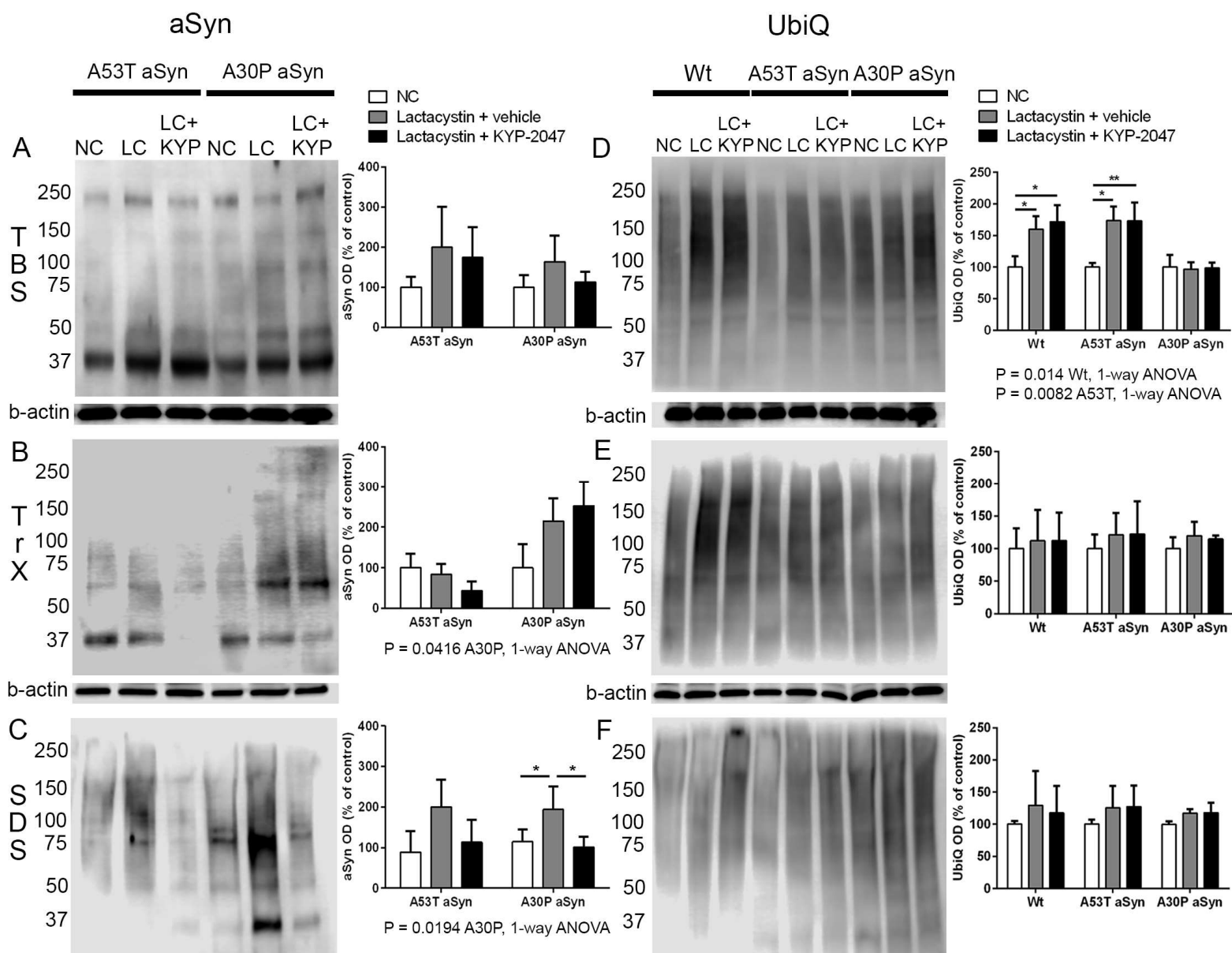


Figure 2A-F. The effect of lactacystin (LC) on $\alpha$-synuclein (aSyn) and ubiquitinated (UbiQ) protein levels in cell culture. $24 \mathrm{~h}$ incubation of LC $(10 \mu \mathrm{M})$ had no effect on TBSsoluble aSyn (A, TBS) but significantly increased the levels of TritonX-soluble (TrX) aSyn oligomers (membrane-bound) on [A30P]aSyn cells while simultaneous incubation with KYP2047 had no effect on this (B). Increase of aSyn oligomers were seen both on SDS-fraction of [A53T]aSyn and [A30P]aSyn cells (C, SDS) and KYP-2047 was able to counter this effect significantly in [A30P]aSyn cells (C). LC did not have effect on aSyn levels on nonoverexpressing SH-SY5Y wild-type cells (Fig. S2 in supplementary materials). LC incubation increased the UbiQ protein levels compared to control only on TBS (soluble) fraction of nonoverexpressing cells and [A53T]aSyn cells (D) but not in [A30P]aSyn cells. LC did not change UbiQ levels in TritonX or SDS fractions (E-F). NC, negative control. * $\mathrm{P}<0.05, * * \mathrm{P}<0.01$, Newman-Keuls post-hoc test, 1-way ANOVA.

\section{The effect of lactacystin and PREP inhibition on proteasomal and autophagy markers}

Ubiquitin levels were significantly increased after lactacystin administration in TBS soluble fraction of Wt (increase of 60-72\%) and [A53T]aSyn cells (increase of 73\%) (Fig. 2D; Wt, $\mathrm{P}=0.0140 ; \mathrm{F}_{2,8}=9.436 ;[\mathrm{A} 53 \mathrm{~T}] \mathrm{aSyn}, \mathrm{P}=0.0082 ; \mathrm{F}_{2,8}=11.87 ; 1$-way ANOVA), and KYP-2047 had no effect on ubiquitin levels. No significant increase was seen in Triton-X or SDS fractions (Fig. 2E-F).

The levels of a protein accumulation marker, p62, was increased in TBS and SDS fractions (Fig. 3A-C; Wt, $\mathrm{P}=0.0379 ; \mathrm{F}_{2,8}=6.792 ; \mathrm{P}<0.05 \mathrm{NC}$ vs. lactacystin +vehicle and $\mathrm{NC}$ vs. lactacystin +KYP-2047, Newman-Keuls post-hoc test; [A53T]aSyn, $\mathrm{P}=0.0143 ; \mathrm{F}_{2,8}=9.369$; TBS fraction: $\mathrm{P}<0.05 \mathrm{NC}$ vs. lactacystin+vehicle and NC vs. lactacystin+KYP-2047, NewmanKeuls post-hoc test; $\left[\mathrm{A} 30 \mathrm{P}\right.$ ] aSyn, $\mathrm{P}=0.0181 ; \mathrm{F}_{2,8}=7.511 ; \mathrm{P}<0.05 \mathrm{NC}$ vs. lactacystin+vehicle and NC vs. lactacystin+KYP-2047, Newman-Keuls post-hoc test). SDS-fraction: Wt, no significance; [A53T]aSyn, $\mathrm{P}=0.0101 ; \mathrm{F}_{2,8}=10.50 ; \mathrm{P}<0.01 \quad \mathrm{NC}$ vs. lactacystin+vehicle, Newman-Keuls post-hoc test; [A30P]aSyn, $\mathrm{P}=0.0011 ; \quad \mathrm{F}_{2,9}=15.99 ; \mathrm{P}<0.0001 \quad \mathrm{NC}$ vs. lactacystin+vehicle, $\mathrm{P}<0.001$ lactacystin+vehicle vs. lactacystin+KYP-2047, Newman-Keuls post-hoc test, 1-way ANOVA). In TrX fraction, p62 was elevated but it did not reach 
significance in OD analysis. Compared to vehicle, KYP-2047 reduced the lactacystin-induced p62 accumulation in SDS fraction of [A30P]aSyn overexpressing cells by $72 \%$ (Fig. $3 \mathrm{C} ; \mathrm{P}<0.01$ lactacystin+vehicle vs. lactacystin+KYP-2047, Newman-Keuls post-hoc test). However, in [A53T]aSyn cells there was no statistically significant difference between NC and lactacystin+KYP-2047 groups, and p62 levels were decreased by KYP-2047 73\% compared to lactacystin+vehicle (Fig. 3C).

Lactacystin incubation increased the levels of LC3BII, an autophagosome marker, (Fig. 3D) suggesting induced autophagy. KYP-2047 increased LC3BII compared to negative control in Wt $(81 \%)$ and [A53T]aSyn $(166 \%)$ cells but not significantly compared to lactacystin and vehicle treatment (Fig 8D; Wt cells: $\mathrm{P}=0.0172 ; \mathrm{F}_{2,10}=6.2731$-way $\mathrm{ANOVA} ; \mathrm{P}<0.05 \mathrm{NC}$ vs. lactacystin+KYP-2047 Newman-Keuls post-hoc test; [A53T]aSyn cells: $\mathrm{P}=0.051 ; \mathrm{F}_{2,13}=3.771$ 1-way ANOVA; P < 0.05 NC vs. lactacystin+KYP-2047 Newman-Keuls post-hoc test). Furthermore, lactacystin had no effect on beclin 1 protein levels (Fig. 3E) but KYP-2047 had a trend to increase beclin1 protein levels (no significance in OD analysis) as seen in our previous study [19]. We also studied the effect of LC and KYP-2047 on parkin, an ubiquitin E3 ligase, which participates in protein ubiquitination and UPS targeting. We observed significantly decreased levels of parkin after LC treatment and KYP-2047 had no effect on parkin levels (Fig. 3F; Wt: $\mathrm{P}=0.0008 ; \mathrm{F}_{2,8}=29.09 ; \mathrm{P}<0.01 \mathrm{NC}$ vs. lactacystin+vehicle, $\mathrm{P}<0.001 \mathrm{NC}$ vs. lactacystin+KYP-2047, Newman-Keuls post-hoc test); [A53T]aSyn, $\mathrm{P}=0.0039 ; \mathrm{F}_{2,8}=16.13$; $\mathrm{P}<0.01 \mathrm{NC}$ vs. lactacystin+vehicle and NC vs. lactacystin+KYP-2047, Newman-Keuls posthoc test; [A30P]aSyn: $\mathrm{P}=0.0021 ; \mathrm{F}_{2,8}=20.43 ; \mathrm{P}<0.01 \mathrm{NC}$ vs. lactacystin+vehicle and $\mathrm{NC}$ vs. lactacystin+KYP-2047, Newman-Keuls post-hoc test, 1-way ANOVA). 
A

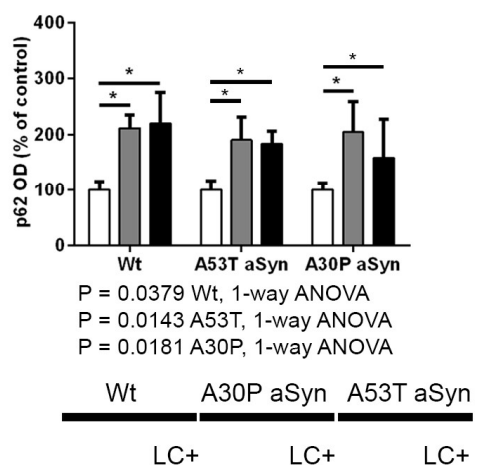

NC LC KYPNC LC KYPNC LC KYP
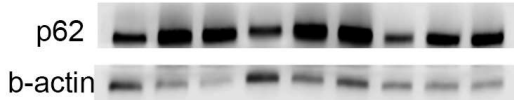

D

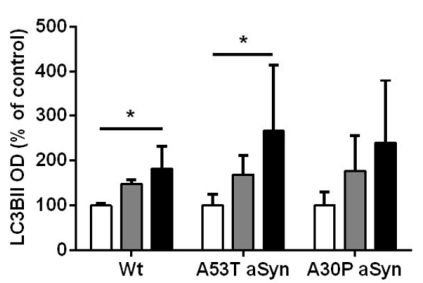

$P=0.0172 \mathrm{Wt}, 1$-way ANOVA

$P=0.051$ A53T, 1-way ANOVA

$\begin{array}{cl}\text { Wt } & \text { A30P aSyn } \\ \text { LC+ }+ \text { LC+ }+ \text { LC+ }\end{array}$

NC LC KYPNC LC KYPNC LC KYP

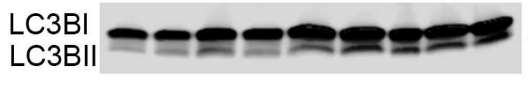

b-actin
B

p62 TrX
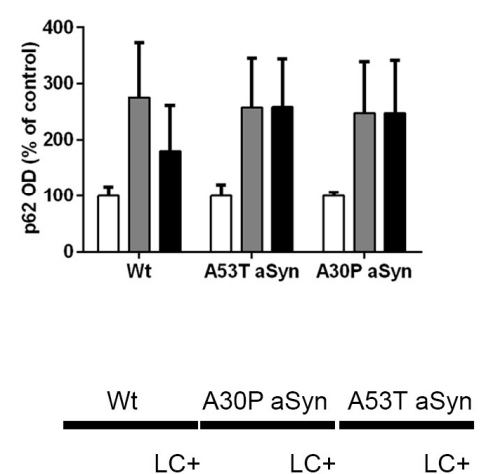

NC LC KYPNC LC KYPNC LC KYP

$-m-m-\infty$

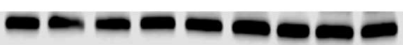

E

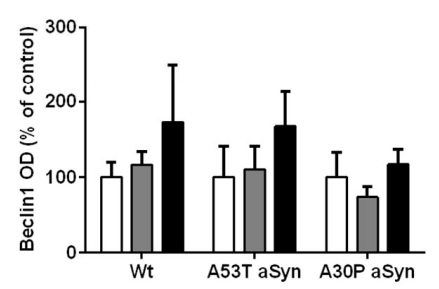

Wt LC+ $_{\text {LC+ }}^{\text {A30P aSyn }} \frac{\text { A53T aSyn }}{\text { LC+ }}$ NC LC KYPNC LC KYPNC LC KYP Beclin1

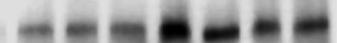

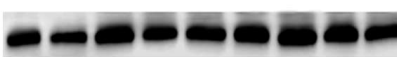

C

p62 SDS

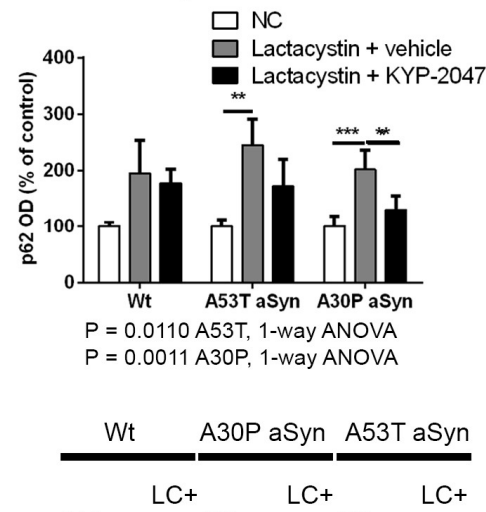
NC LC KYPNC LC KYPNC LC KYP
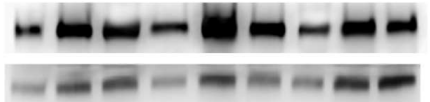

F

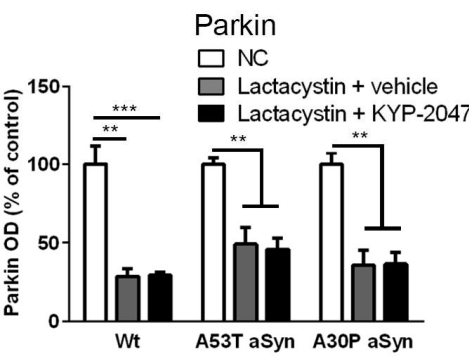

$P=0.0008 \mathrm{Wt}$, 1-way ANOVA

$P=0.0039$ A53T, 1-way ANOVA

$P=0.0021 \mathrm{A30P}, 1-$ way ANOVA

$\frac{\text { Wt }}{\text { LC+ }+ \text { A30P aSyn }} \frac{\text { A53T aSyn }}{\text { LC+ }}$

NC LC KYPNC LC KYPNC LC KYP

Parkin $\square-\infty+\infty$

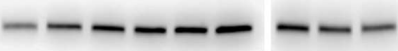

Figure 3A-E. The effect of a proteasome inhibitor, lactacystin (LC), incubation on autophagy markers in cell culture. LC (10 $\mu \mathrm{M}, 24 \mathrm{~h})$ incubation induced significant increase of a protein accumulation marker, p62, in TBS and SDS fractions (A-C). KYP-2047, a prolyl oligopeptidase inhibitor, significantly blocked the LC induced increase of p62 in SDS fraction of [A30P]aSyn cells (C). LC incubation caused an increase on the autophagosome marker, LC3BII (D) but it was not statistically significant in optical density (OD) analysis. In wild-type (Wt) and [A53T]aSyn cells, KYP-2047 had significant effect on LC3BII compared to vehicle treatment. LC did not alter beclin1 levels (E) while KYP-2047 had a trend to increase beclin1 amounts. Parkin1 levels were significantly decreased by LC (F) and KYP-2047 did not have effect on this. ${ }^{*} \mathrm{P}<0.05, * * \mathrm{P}<0.01, * * * \mathrm{P}<0.001$, Newman-Keuls post-hoc test, 1-way ANOVA.

\section{Discussion}


In this study, we have shown that a PREP inhibitor, KYP-2047, effectively attenuates the toxic effects of lactacystin in mutated aSyn overexpressing cells by decreasing the levels of accumulated mutated aSyn.

After aSyn and its aggregation has been identified as one of the key players in PD pathophysiology, also proteolytic balance has raised interest in PD research since decreased proteasomal activities [9] and reduced lysosomal markers (for review, see [27]) have been found from PD patients. UPS and chaperone-mediated autophagy are mainly responsible for degradation of soluble aSyn. However, misfolding and aggregation of aSyn leads to protein accumulation, deficits on UPS and chaperone-mediated autophagy, and thereafter to induction of autophagy, which can also degrade oligomeric forms of aSyn (for reviews, see [28]).

Several studies show that activating autophagy may be beneficial to neuronal survival in aSyn aggregation models (for review, see [21]). We showed in our previous study that a PREP inhibitor, KYP-2047, enhanced autophagy via beclin1 dependent mechanism and decreased the amount of HMW aSyn in cell culture and in the brain of aSyn transgenic mouse $[19,20]$. Therefore, we wanted to test if PREP inhibition can overcome the aSyn aggregation induced by proteasomal deficit in a cellular model. Our results show that the inhibition of proteasomal activity in mutated aSyn overexpressing cells resulted in a dose-dependent reduction of cell viability and increase in aSyn oligomerization whereas cells without mutated aSyn overexpression were more resistant to lactacystin-induced cell death, supporting the significance of aSyn aggregation in lactacystin induced toxicity. Treatment of aSyn overexpressing cell lines with KYP-2047 significantly improved cell survival together with reduced HMW aSyn oligomers and SDS-insoluble p62, suggesting induced autophagy. Interestingly, [A30P] aSyn overexpressing cells showed accumulation also in $\operatorname{TrX}$ fraction and KYP-2047 did not have any effect on this. When considering that A30P[aSyn] is more prone for membrane-associated aggregation than other forms of aSyn [6], this suggests that PREP 
inhibitors are effective towards aggregation occurring in cytosol. Although we did not see significant increase in LC3BII and Beclin1 in this study, these markers showed increasing trend, and taken together with the decrease in SDS-soluble p62, a protein accumulation marker, the effects of KYP-2047 seem specific for insoluble aggregated proteins, emphasizing the role for autophagy. Our current and previous results suggest that PREP regulates PI3K class 3 mediated autophagy pathway, and the exact role of PREP on this pathway needs to be studied further.

We saw increased ubiquitin protein levels only in soluble fraction of [A53T]aSyn overexpressing cells. Increased ubiquitin load indicates declined proteasomal activity, and is also associated to Lewy bodies of PD. However, lactacystin does not seem to induce accumulation of insoluble ubiquitin positive proteins. This might be explained by our observation of reduced parkin protein amount after lactacystin treatment. Parkin is important for ubiquitinylation of proteins which has been shown to be downregulated after lactacystin also in previous study [29]. KYP-2047 did not alter the ubiquitin levels but reduced p62 and aSyn oligomers in SDS-fraction suggest that PREP inhibition does not affect directly on degradation of soluble proteins but is more effective in inducing autophagy and clearance of insoluble proteins and aggregates.

\section{Conclusions}

Our results show that aSyn overexpression promotes lactacystin-induced toxicity and KYP2047 protects cells against cell death by decreasing insoluble mutated aSyn oligomers and p62 levels. Several diseases with protein accumulation, including neurodegenerative diseases, would benefit from autophagy enhancement but there have been various problems, such as specificity and toxicity, in developing autophagy enhancers for clinical use [30]. However, 
PREP inhibition may offer novel means to induce autophagy and treat synucleinopathies as well as other protein aggregating diseases

\section{Acknowledgements}

This work was supported by grants from the Academy of Finland (267788 and 2737991), University of Helsinki research grants, Jane and Aatos Erkko Foundation and from the Sigrid Juselius Foundation. Sponsors did not participate in study design, or collection, analysis and interpretation of data or writing the report.

\section{References}

[1] M.G. Spillantini, M.L. Schmidt, V.M. Lee, J.Q. Trojanowski, R. Jakes, M. Goedert, Alpha-synuclein in Lewy bodies, Nature 388 (1997) 839-840.

[2] K.C. Luk, V.M. Kehm, B. Zhang, P. O'Brien, J.Q. Trojanowski, V.M. Lee, Intracerebral inoculation of pathological alpha-synuclein initiates a rapidly progressive neurodegenerative alpha-synucleinopathy in mice, J. Exp. Med. 209 (2012) 975-986.

[3] E. Angot, J.A. Steiner, C.M. Lema Tome, P. Ekstrom, B. Mattsson, A. Bjorklund, P. Brundin, Alpha-synuclein cell-to-cell transfer and seeding in grafted dopaminergic neurons in vivo, PLoS One 7 (2012) e39465.

[4] A. Surguchov, Molecular and cellular biology of synucleins, Int. Rev. Cell. Mol. Biol. 270 (2008) 225-317.

[5] W. Xu, L. Tan, J.T. Yu, Link between the SNCA gene and parkinsonism, Neurobiol. Aging 36 (2015) 1505-1518.

[6] H.A. Lashuel, C.R. Overk, A. Oueslati, E. Masliah, The many faces of alpha-synuclein: from structure and toxicity to therapeutic target, Nat. Rev. Neurosci. 14 (2013) 38-48.

[7] E. Emmanouilidou, L. Stefanis, K. Vekrellis, Cell-produced alpha-synuclein oligomers are targeted to, and impair, the 26S proteasome, Neurobiol. Aging 31 (2010) 953-968.

[8] H. Snyder, K. Mensah, C. Theisler, J. Lee, A. Matouschek, B. Wolozin, Aggregated and monomeric alpha-synuclein bind to the S6' proteasomal protein and inhibit proteasomal function, J. Biol. Chem. 278 (2003) 11753-11759. 
[9] K.S. McNaught, R. Belizaire, O. Isacson, P. Jenner, C.W. Olanow, Altered proteasomal function in sporadic Parkinson's disease, Exp. Neurol. 179 (2003) 38-46.

[10] A.M. Cuervo, L. Stefanis, R. Fredenburg, P.T. Lansbury, D. Sulzer, Impaired degradation of mutant alpha-synuclein by chaperone-mediated autophagy, Science 305 (2004) 1292-1295.

[11] A.R. Winslow, C.W. Chen, S. Corrochano, A. Acevedo-Arozena, D.E. Gordon, A.A. Peden, M. Lichtenberg, F.M. Menzies, B. Ravikumar, S. Imarisio, S. Brown, C.J. O'Kane, D.C. Rubinsztein, alpha-Synuclein impairs macroautophagy: implications for Parkinson's disease, J. Cell Biol. 190 (2010) 1023-1037.

[12] W. Xie, X. Li, C. Li, W. Zhu, J. Jankovic, W. Le, Proteasome inhibition modeling nigral neuron degeneration in Parkinson's disease, J. Neurochem. 115 (2010) 188-199.

[13] E. Bentea, A. Van der Perren, J. Van Liefferinge, A. El Arfani, G. Albertini, T. Demuyser, E. Merckx, Y. Michotte, I. Smolders, V. Baekelandt, A. Massie, Nigral proteasome inhibition in mice leads to motor and non-motor deficits and increased expression of Ser129 phosphorylated alpha-synuclein, Front. Behav. Neurosci. 9 (2015) 68.

[14] H.J. Rideout, K.E. Larsen, D. Sulzer, L. Stefanis, Proteasomal inhibition leads to formation of ubiquitin/alpha-synuclein-immunoreactive inclusions in PC12 cells, J. Neurochem. 78 (2001) 899-908.

[15] M.H. Savolainen, X. Yan, T.T. Myohanen, H.J. Huttunen, Prolyl Oligopeptidase Enhances alpha-synuclein Dimerization via Direct Protein-Protein Interaction, J. Biol. Chem. 290 (2015) 5117-5126.

[16] I. Brandt, M. Gerard, K. Sergeant, B. Devreese, V. Baekelandt, K. Augustyns, S. Scharpe, Y. Engelborghs, A.M. Lambeir, Prolyl oligopeptidase stimulates the aggregation of alpha-synuclein, Peptides 29 (2008) 1472-1478.

[17] M.J. Hannula, T.T. Myohanen, J. Tenorio-Laranga, P.T. Mannisto, J. Arturo GarciaHorsman, Prolyl oligopeptidase colocalizes with alpha-synuclein, beta-amyloid, tau protein and astroglia in the post-mortem brain samples with Parkinson's and Alzheimer's diseases, Neuroscience 242 (2013) 140-150.

[18] T.T. Myohanen, M.J. Hannula, R. Van Elzen, M. Gerard, P. Van Der Veken, J.A. Garcia-Horsman, V. Baekelandt, P.T. Mannisto, A.M. Lambeir, A prolyl oligopeptidase inhibitor, KYP-2047, reduces alpha-synuclein protein levels and aggregates in cellular and animal models of Parkinson's disease, Br. J. Pharmacol. 166 (2012) 1097-1113.

[19] M.H. Savolainen, C.T. Richie, B.K. Harvey, P.T. Mannisto, K.A. Maguire-Zeiss, T.T. Myohanen, The beneficial effect of a prolyl oligopeptidase inhibitor, KYP-2047, on alphasynuclein clearance and autophagy in A30P transgenic mouse, Neurobiol. Dis. 68C (2014) 115.

[20] L. Dokleja, M.J. Hannula, T.T. Myohanen, Inhibition of prolyl oligopeptidase increases the survival of alpha-synuclein overexpressing cells after rotenone exposure by reducing alpha-synuclein oligomers, Neurosci. Lett. 583C (2014) 37-42. 
[21] D.C. Rubinsztein, C.F. Bento, V. Deretic, Therapeutic targeting of autophagy in neurodegenerative and infectious diseases, J. Exp. Med. 212 (2015) 979-990.

[22] E.M. Jarho, J.I. Venalainen, J. Huuskonen, J.A. Christiaans, J.A. Garcia-Horsman, M.M. Forsberg, T. Jarvinen, J. Gynther, P.T. Mannisto, E.A. Wallen, A cyclopent-2-enecarbonyl group mimics proline at the P2 position of prolyl oligopeptidase inhibitors, J. Med. Chem. 47 (2004) 5605-5607.

[23] A.J. Jalkanen, J.J. Hakkarainen, M. Lehtonen, T. Venalainen, T.M. Kaariainen, E. Jarho, M. Suhonen, M.M. Forsberg, Brain pharmacokinetics of two prolyl oligopeptidase inhibitors, JTP-4819 and KYP-2047, in the rat, Basic Clin. Pharmacol. Toxicol. 109 (2011) 443-451.

[24] A.J. Jalkanen, T.P. Piepponen, J.J. Hakkarainen, I. De Meester, A.M. Lambeir, M.M. Forsberg, The effect of prolyl oligopeptidase inhibition on extracellular acetylcholine and dopamine levels in the rat striatum, Neurochem. Int. 60 (2012) 301-309.

[25] M. Gerard, A. Deleersnijder, V. Daniels, S. Schreurs, S. Munck, V. Reumers, H. Pottel, Y. Engelborghs, C. Van den Haute, J.M. Taymans, Z. Debyser, V. Baekelandt, Inhibition of FK506 binding proteins reduces alpha-synuclein aggregation and Parkinson's disease-like pathology, J. Neurosci. 30 (2010) 2454-2463.

[26] T. Fagerqvist, V. Lindstrom, E. Nordstrom, A. Lord, S.M. Tucker, X. Su, C. Sahlin, A. Kasrayan, J. Andersson, H. Welander, T. Nasstrom, M. Holmquist, H. Schell, P.J. Kahle, H. Kalimo, C. Moller, P. Gellerfors, L. Lannfelt, J. Bergstrom, M. Ingelsson, Monoclonal antibodies selective for alpha-synuclein oligomers/protofibrils recognize brain pathology in Lewy body disorders and alpha-synuclein transgenic mice with the disease-causing A30P mutation, J. Neurochem. 126 (2013) 131-144.

[27] T. Moors, S. Paciotti, D. Chiasserini, P. Calabresi, L. Parnetti, T. Beccari, W.D. van de Berg, Lysosomal Dysfunction and alpha-Synuclein Aggregation in Parkinson's Disease: Diagnostic Links, Mov. Disord. 31 (2016) 791-801.

[28] D. Ebrahimi-Fakhari, L. Wahlster, P.J. McLean, Protein degradation pathways in Parkinson's disease: curse or blessing, Acta Neuropathol. 124 (2012) 153-172.

[29] A. Koch, K. Lehmann-Horn, J.C. Dachsel, T. Gasser, P.J. Kahle, C.B. Lucking, Proteasomal inhibition reduces parkin mRNA in PC12 and SH-SY5Y cells, Parkinsonism Relat. Disord. 15 (2009) 220-225.

[30] B. Levine, M. Packer, P. Codogno, Development of autophagy inducers in clinical medicine, J. Clin. Invest. 125 (2015) 14-24. 
Supplementary material for manuscript "Prolyl oligopeptidase inhibition attenuates the toxicity of a proteasomal inhibitor, lactacystin, in the alpha-synuclein overexpressing cell culture"
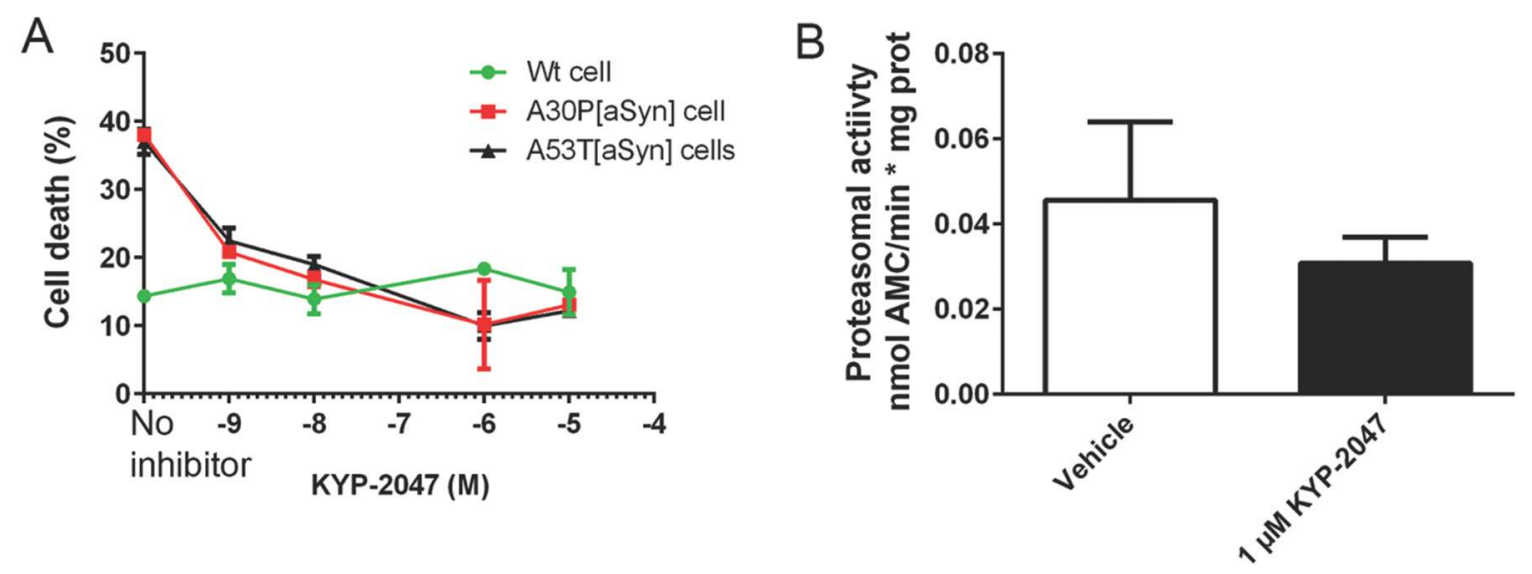

Figure S1A-B. The dose-response curve of KYP-2047 on cell viability and effect of KYP-2047 on cell proteasomal activity. Concentrations between $10^{-9}$ to $10^{-5} \mathrm{M}$ of KYP-2047 were tested against $10 \mu \mathrm{M}$ lactacystin toxicity in LDH test. Some protection by KYP-2047 was seen already at $1 \mathrm{nM}$ but the most effective dose was $1 \mu \mathrm{M}$ and it was chosen for experiments (A). $1 \mu \mathrm{M} \mathrm{KYP}-2047$ did not have significant effect on cell proteasomal activity in non-overexpressing SH-SY5Y cells (B). 


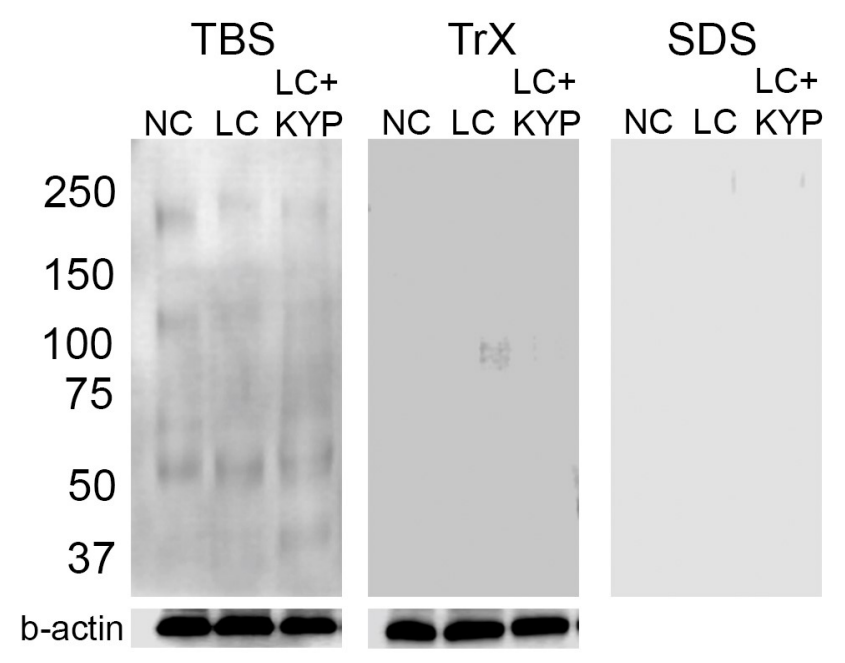

Figure S2. The effect of lactacystin (LC) on $\alpha$-synuclein (aSyn) levels in wild-type (Wt) nonaSyn overexpressing cell culture. $24-\mathrm{h}$ incubation of Wt cells with $10 \mu \mathrm{M}$ LC or LC $+1 \mu \mathrm{M}$ KYP2047 (LC+KYP) did not show any detectable high-molecular weight oligomers in TBS-soluble, TritonX-soluble ( $\mathrm{TrX}$ ) or SDS-fraction (SDS) compared to negative control (NC). Beta-actin served as a loading control. 


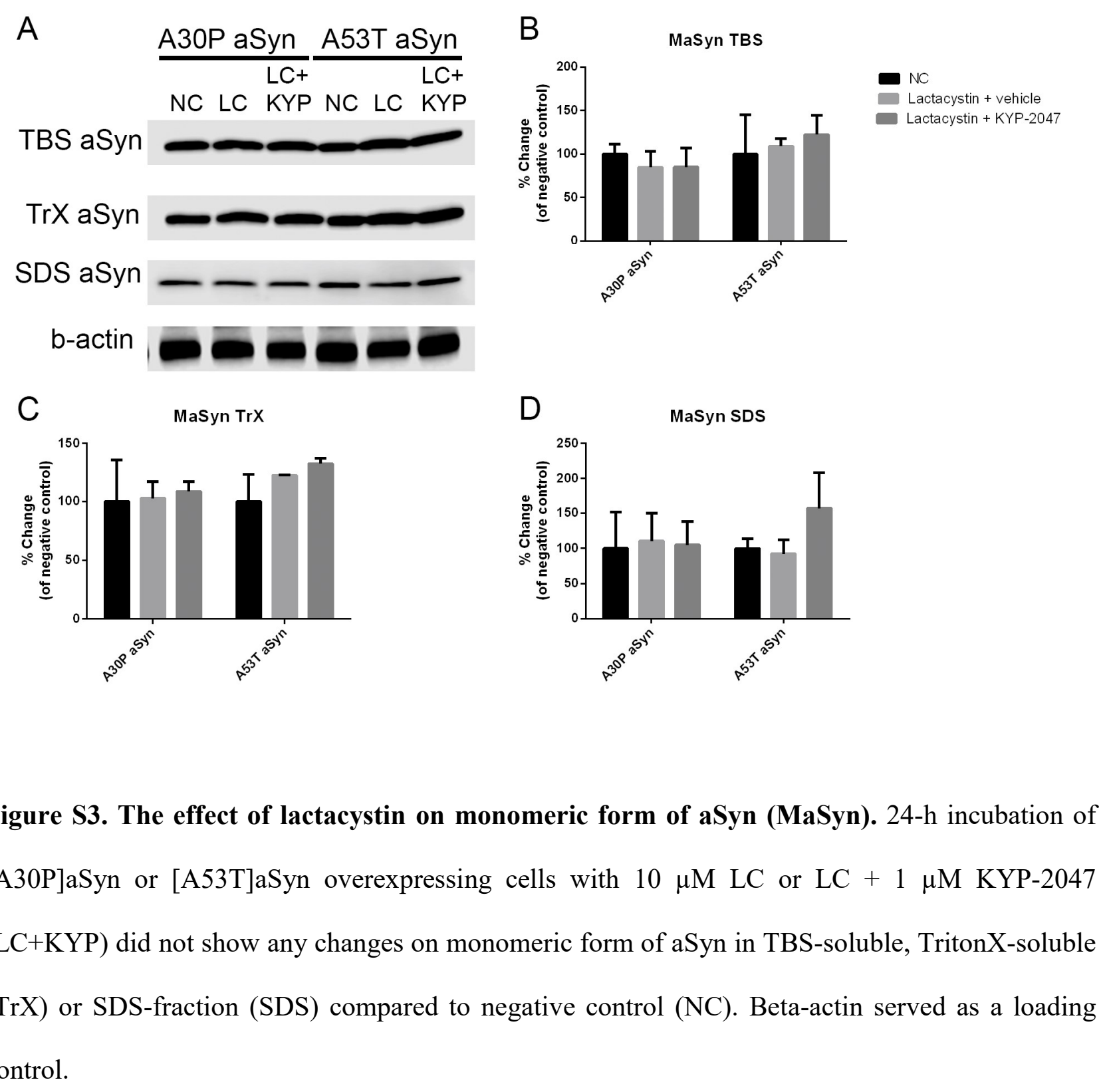

